\title{
Gastrocnemius and tibialis anterior neuromuscular modification recruitment during postural standing in people living with HIV
}

\author{
Martin G. Rosario \\ Texas Woman's University's School of Physical Therapy, United States
}

\begin{abstract}
Introduction: A known repercussion of human immunodeficiency virus (HIV) is an alteration of gait and balance, which increases the risk of fall-related injuries for those living with the disease. Muscle weakness and neurocognitive alterations can lead to distorted postural strategies in people living with HIV (PLHIV); however, the precise neuromuscular mechanism leading to this impaired postural instability remains unknown. The aim of this study was to examine the neuromuscular recruitment of lower extremities during single and dual-cognitive balance tasks among PLHIV.

Material and methods: Fifty adults participated in this study, of whom 19 were diagnosed HIV-positive and 31 were HIV-negative. Each participant had surface electromyography electrodes placed on the tibialis anterior (TA) and gastrocnemius (GA) muscles of their dominant leg before being instructed to stand in a bi-pedal posture on a balance foam pad for 15 seconds per task. Four single balance tasks and four dual-cognitive balance tasks were performed on the foam pad.

Results: Significant modifications in delayed and prolonged neuromuscular activation recruitment were found in both the GA and TA during single tasks, whereas dual cognitive tasks presented comparable results between the HIV group and the control group.

Conclusions: Asymptomatic PLHIV exhibit altered neuromuscular recruitment while standing, which suggest a correlation between this evident increase in modification and a localized, peripheral issue or miscommunication between the central and peripheral nervous systems. This research highlights the necessity of conducting lower extremity neuromuscular assessments in all HIV-positive individuals.
\end{abstract}

\author{
HIV AIDS Rev 2020; 19, 4: 260-266 \\ DOI: https://doi.org/10.5114/hivar.2020.101633
}

Key words: postural stability, balance, HIV, neuromuscular modifications.

\section{Introduction}

According to the HIV Surveillance Report of the Center for Disease Control and Prevention from 2019, human immunodeficiency virus (HIV) affects 36.9 million people world- wide, of whom 1.1 million are exclusively in the United States (US) [1]. Within the US, Texas and Puerto Rico are among the locations with the highest incidence rate, which have continued to increase over the last few years. HIV reproduces within the brain's immune cells, leading to substantial cognitive and
Address for correspondence: Dr. Martin G. Rosario, Texas Woman's University's School of Physical Therapy, 5500 Southwestern Medical Avenue, Dallas, TX 75235,

United States, e-mail: mrosariol@twu.edu
Article history:

Received: 09.04.2020

Received in revised form: 09.05.2020

Accepted: 14.05.2020

Available online: 30.11 .2020
International Journal of HIV-Related Problems

HIV \& AIDS

R e vi e w 
motor disturbances that hinder everyday activities and are associated with a lower quality of life; therefore, heightening distress for both caretakers and healthcare system. Despite the advancement in antiretroviral therapy (ART) to fight the replication of the virus, HIV still destructively affects gait and balance, leading HIV-positive individuals to be more susceptible to falls, trauma, and premature mortality [2-4].

Among the issues related to HIV, $41 \%$ of people living with HIV (PLHIV) who are over 50 years of age experienced a fall in the last year [5]. Similarly, 38\% of individuals with this virus reported having balance impairments, citing obesity as one of the justifications for being at risk for falls, when associated with reduced performance of dynamic balance tests [6]. HIV-positive patients, with a body mass index (BMI) within the obese range, experience additional alterations in balance and gait variables as compared to those within the underweight BMI range. From this, the clinical implication is clear that PLHIV have a natural tendency towards obesity, causing them to carry a significantly higher risk for balance and gait impairments in comparison to those with a normal or underweight BMI, or those who are HIV-negative [7].

Due to advancements in medications for HIV, PLHIV are proceeding to have prolonged life expectancies. While the value of extended lifespans are invaluable, it comes with one distinct complication: the longer the time is that people live with HIV, the greater the danger becomes of PLHIV acquiring neuromotor and/ or cognitive difficulties related to a long-term effects of the illness [10]. For instance, older men infected with HIV are 57\% more likely to have considerably slower gait speed, a variable that is predictive of accelerated aging among this population, which also includes a prompt rate of functional deterioration. On average, the decline in gait speed emerges considerably more $(0.025 \mathrm{~m} / \mathrm{s})$ per year in men over the age of 50 who are HIV-positive than those who are HIV-negative [10].

Increased attention has been focused on how HIV causes neuromotor alterations and impairments, specifically in regard to gait and balance. One premise involves HIV medications, as they tend to damage the mitochondria, which affects muscle function, ultimately leading to sarcopenia and osteoporosis. Consequently, sarcopenia and osteoporosis could be partly responsible for the gait and balance deficits seen in this population due to their impact on body strength and balance strategies. Diminished power in the lower extremity musculature leads to an increased co-activation of leg musculature. Moreover, improper lower limb co-activation may lead to a hip strategy shifting and, therefore, postural sway alterations [6].

Another suggested complication responsible for balance and gait impairments in PLHIV is an inappropriate selection of compensatory strategies for postural correction due to inaccurate processing of balance information in the central nervous system (CNS) [6]. Likewise, the disease might directly impact vestibular input, leading to increased frequency of movement and increased postural sway [15].

Due to the above-mentioned findings, the objective of this study was two-fold. Firstly, to distinguish lower limb neuromuscular recruitment activation patterns while standing among physically active PLHIV in comparison to HIV-negative, physically active individuals. Secondly, as some authors investigated neurocognitive consequences of long-term living with HIV and how HIV affects the balance, our study intended to explore how adding cognitive dual-tasks would modify lower limb muscle recruitment during standing balance in PLHIV. Ultimately, this study strives to characterize the neuromuscular control that affects standing postural stability to recommended targeted interventions for PLHIV.

Compared to the control group, we hypothesize that PLHIV would exhibit: 1) evident neuromuscular recruitment alterations in the tibialis anterior and gastrocnemius muscles during standing postural control tasks; 2) as the balance tasks increase in complexity and incorporate dual-cognitive tasks, modifications in the neuromuscular alteration recruitment pattern of the tibialis anterior and gastrocnemius muscles would increase.

\section{Material and methods}

\section{Participants}

This study was advertised through pamphlets and word of mouth at both the Dallas and Puerto Rico locations. Those interested in participating in this research, contacted the study team before being informed on the requirements of the study and the necessary criteria to participate. All of the study participants signed an informed consent, which was approved by an institution's IRB.

\section{Control group}

The control group consisted of young, healthy, HIV-negative adults. A total of 31 healthy participants ( 7 male, 24 female; average age, $25.07 \pm 3.46$ years; average BMI, 23.92) were recruited from the Texas Woman's University's (TWU) T. Boone Pickens Institute of Health Sciences Dallas Campus as well as its surrounding communities. Data collection for the control group was performed at the TWU. The control group underwent the same screening and balance protocol as the HIV group as well as comparable inclusion criteria, sex, and age.

\section{HIV group}

The portion of this study conducted on the HIV group was managed in San Juan, Puerto Rico, at a Hispanic/Latino Rehabilitation Clinic for HIV patients called "La Perla de Gran Precio" (LPGP). The LPGP is a fitness and rehabilitation center that specializes in aiding people living with HIV, with a variety of medical complications, backgrounds, and profiles. For this study, the experimental HIV group consisted of 19 participants diagnosed with HIV (14 male, 5 female; average age, $56.06 \pm 7.7$ years; average BMI, $25.1 \pm 5.7)$, with an average CD4 count of 739.53 \pm 303.2 (23 years of HIV diagnosis). All participants in the HIV group were of Hispanic-Latino origin. The consent 
Table 1. Demographic data of all participants

\begin{tabular}{l|c|c}
\hline Characteristics & Control, $n=31$ & HIV participants, $n=19$ \\
\hline Age (years) & $25.07 \pm 3.46$ & $56.06 \pm 7.7$ \\
\hline \begin{tabular}{l} 
Gender \\
\hline Male
\end{tabular} & 7 & 5 \\
\hline Female & 24 & 14 \\
\hline CD4, mean & - & $739.53 \pm 303.2$ \\
\hline BMI, mean & 23.92 & $25.1 \pm 5.7$ \\
\hline
\end{tabular}

form granted access to their medical records, such as HIV status, CD4+ cell counts, and different prerequisites necessary to participate in this investigation.

\section{Criteria}

Inclusion criteria for both the control group and HIV group were as follow:

1) age within the range of $25-80$ years;

2) ability to walk without an assistive device;

3) ability to tolerate standing for at least 30 minutes;

4) have a stable cardiorespiratory system;

5) ability to successfully perform a 5 times sit-to-stand test. In addition to those above, the specific inclusion criteria for the HIV group included being diagnosed with HIV and having CD4+ cell count levels above 200 cells/ $\mu$ l.

Exclusion criteria for both the control group and HIV group included:

1) severe balance impairments assessed by a Romberg test;

2) severe visual acuity problems that were not treated;

3) falls occurring over the last six months;

4) back or lower extremity lesions or surgeries in the past six months;

5) use of prescriptions that cause drowsiness 24 hours prior to participating in this study;

6) women that are pregnant or suspect they might be pregnant. Each subject was evaluated through an interview process and in the case of the HIV group, their medical records were reviewed for the inclusion and exclusion criteria assessment.

\section{Equipment}

For this investigation, electromyography (EMG) electrodes were used to collect on time data of activity and patterns from the tibialis anterior (TA) and gastrocnemius (GA) muscles of the dominant leg. The variables of interest recorded in this study included onset of the highest muscular activation (in seconds), time at the peak activation (in seconds), time of the muscle activation decay (in seconds), and duration of the muscle activity (in seconds).

\section{Procedures}

After all participants signed the approved informed consent, researchers collected demographic information and baseline vitals, such as blood pressure, pulse, and $\mathrm{SpO}_{2}$ levels (Table 1). Participants who wore glasses were identified and kept them on during testing. The maximum time commitment for each participant was 30 minutes, which included both screening and balance testing protocols. Prior to collecting data, participants identified their dominant leg. If participants were unsure of their dominant leg, they were given a perturbation from behind, and whichever leg was used for their stepping strategy was identified as their dominant leg. If necessary, areas of the dominant leg were shaved with a non-electric razor to attach and secure the placement of EMG electrodes. An EMG surface electrode was placed over the TA and GA of the dominant leg.

\section{Balance assessment}

While wearing the EMG sensors, each participant was instructed to stand in a static bi-pedal posture. After establishing baseline estimations, a 3 -inch foam pad (2.4 inches thick, 15.5 inches long, and 12.5 inches wide) was placed on the floor to be added to eight balance tasks. Participants resumed the same static bi-pedal posture on top of the foam pad, and each task was recorded for 15 seconds. Balance tasks were divided into four non-cognitive tasks and four dual-cognitive tasks. For each task, including the baseline measurement, participants were instructed to fixate their gaze on an orange square on the wall, located 10 feet away from them.

\section{Balance protocol}

All balance tasks were performed on the foam pad placed on the floor. The four non-cognitive tasks were: 1) eyes open (EO FOAM), 2) eyes closed (EC FOAM), 3) eyes open while moving the head up and down to the cadence of a $60 \mathrm{bpm}$ metronome (EO HUD), and 4) eyes closed while moving the head up and down to the cadence of a $60 \mathrm{bpm}$ metronome (EC HUD). Following this, the four dualcognitive ( $\operatorname{cog}$ ) tasks were conducted in an identical manner to the four non-cognitive tasks, with one addition of counting backward from 100 by 3 during each task.

\section{Data analysis}

For the EMG analysis, we identified the highest peak of muscle activity for both the TA and GA for each of the eight tasks. The time, in seconds, at the initiation of each peak was determined as the time for onset. The time to peak was established as the time to the highest peak of activity. The time at the end of the muscle activation or from the highest activation was identified as the decay. Finally, the muscle activity duration was measured as the distance between onset and decay for both the TA and GA.

\section{Results}

The time for onset, time to peak, decay, and duration variables were analyzed by SPSS (Statistical Package for 
Table 2. Electromyographic onset, time to peak, duration, and decay of gastrocnemius during balance tasks. Results of multivariate analysis of variance (MANOVA) performed between asymptomatic HIV group and control group. Significance level set at $p \leq 0.05$

\begin{tabular}{|c|c|c|c|}
\hline $\begin{array}{l}\text { Gastrocnemius } \\
\text { Task/Variables (s) }\end{array}$ & $\begin{array}{l}\text { Control } \\
(n=19)\end{array}$ & $\begin{array}{l}\text { Asymptomatic } \\
\text { HIV }(n=18)\end{array}$ & $p$-value \\
\hline \multicolumn{4}{|l|}{ EO Firm } \\
\hline Onset & $8.00 \pm 4.90$ & $6.80 \pm 4.07$ & 0.34 \\
\hline Time to peak & $0.60 \pm 0.60$ & $0.50 \pm 0.35$ & 0.51 \\
\hline Duration & $1.20 \pm 0.69$ & $1.04 \pm 0.62$ & 0.36 \\
\hline Decay & $0.57 \pm 0.37$ & $0.49 \pm 0.39$ & 0.47 \\
\hline \multicolumn{4}{|l|}{ EO Foam } \\
\hline Onset & $7.80 \pm 5.60$ & $11.30 \pm 5.50$ & 0.05 \\
\hline Time to peak & $0.49 \pm 0.44$ & $0.57 \pm 0.31$ & 0.50 \\
\hline Duration & $1.04 \pm 0.73$ & $1.28 \pm 0.72$ & 0.24 \\
\hline Decay & $0.55 \pm 0.45$ & $0.71 \pm 0.53$ & 0.21 \\
\hline \multicolumn{4}{|l|}{ EC Foam } \\
\hline Onset & $8.30 \pm 4.50$ & $10.48 \pm 5.50$ & 0.11 \\
\hline Time to peak & $0.69 \pm 0.52$ & $0.69 \pm 0.41$ & 0.99 \\
\hline Duration & $1.30 \pm 0.74$ & $1.60 \pm 0.90$ & 0.18 \\
\hline Decay & $0.61 \pm 0.36$ & $0.91 \pm 0.72$ & 0.05 \\
\hline \multicolumn{4}{|l|}{ EO HUD } \\
\hline Onset & $7.20 \pm 4.50$ & $10.30 \pm 5.70$ & 0.05 \\
\hline Time to peak & $0.50 \pm 0.40$ & $0.67 \pm 0.57$ & 0.22 \\
\hline Duration & $1.11 \pm 0.66$ & $1.15 \pm 0.68$ & 0.84 \\
\hline Decay & $0.61 \pm 0.39$ & $0.49 \pm 0.27$ & 0.21 \\
\hline \multicolumn{4}{|l|}{ EC HUD } \\
\hline Onset & $6.90 \pm 4.90$ & $11.60 \pm 5.20$ & 0.001 \\
\hline Time to peak & $0.50 \pm 0.30$ & $0.70 \pm 0.60$ & 0.12 \\
\hline Duration & $1.20 \pm 0.60$ & $1.50 \pm 0.75$ & 0.15 \\
\hline Decay & $0.70 \pm 0.43$ & $0.73 \pm 0.31$ & 0.51 \\
\hline \multicolumn{4}{|l|}{ EO COG } \\
\hline Onset & $9.70 \pm 5.30$ & $13.10 \pm 6.10$ & 0.05 \\
\hline Time to peak & $0.53 \pm 0.41$ & $0.44 \pm 0.20$ & 0.35 \\
\hline Duration & $1.07 \pm 0.67$ & $0.97 \pm 0.41$ & 0.58 \\
\hline Decay & $0.54 \pm 0.42$ & $0.53 \pm 0.34$ & 0.99 \\
\hline \multicolumn{4}{|l|}{ EC COG } \\
\hline Onset & $7.30 \pm 4.30$ & $9.30 \pm 4.90$ & 0.17 \\
\hline Time to peak & $0.51 \pm 0.53$ & $0.52 \pm 0.45$ & 0.94 \\
\hline Duration & $1.10 \pm 0.94$ & $1.09 \pm 0.63$ & 0.85 \\
\hline Decay & $0.63 \pm 0.59$ & $0.58 \pm 0.29$ & 0.72 \\
\hline
\end{tabular}

Social Sciences) version 25, using a multivariate analysis of variance (MANOVA) examination for the differences between the control group and HIV group. We divided the balance examination into single tasks and dual-cognitive tasks.
Table 2. Cont.

\begin{tabular}{l|c|c|c}
\hline $\begin{array}{l}\text { Gastrocnemius } \\
\text { Task/Variables (s) }\end{array}$ & $\begin{array}{c}\text { Control } \\
(n=19)\end{array}$ & $\begin{array}{c}\text { Asymptomatic } \\
\text { HIV }(n=18)\end{array}$ & $p$-value \\
\hline \begin{tabular}{l} 
EO HUD COG \\
\hline Onset
\end{tabular} & $8.90 \pm 5.60$ & $8.09 \pm 5.00$ & 0.59 \\
\hline Time to peak & $0.57 \pm 0.56$ & $0.51 \pm 0.34$ & 0.66 \\
\hline Duration & $1.30 \pm 1.21$ & $1.07 \pm 0.58$ & 0.41 \\
\hline Decay & $0.75 \pm 0.88$ & $0.57 \pm 0.31$ & 0.40 \\
\hline EC HUD COG & $8.50 \pm 5.60$ & $9.50 \pm 6.60$ & 0.56 \\
\hline Onset & $0.70 \pm 1.01$ & $0.53 \pm 0.33$ & 0.47 \\
\hline Time to peak & $1.20 \pm 1.20$ & $1.12 \pm 0.67$ & 0.79 \\
\hline Duration & $0.52 \pm 0.41$ & $0.54 \pm 0.42$ & 0.54 \\
\hline Decay &
\end{tabular}

During a single task, the slow neuromuscular recruitment $(p<0.05)$ was displayed by a longer onset time during EO FOAM (control, $7.8 \pm 5.6 \mathrm{~s}$; HIV, $11.3 \pm 5.5 \mathrm{~s}$ ) and EC FOAM (control, $8.3 \pm 4.5 \mathrm{~s}$; HIV, $10.48 \pm 5.5 \mathrm{~s}$ ) for the GA muscle, as seen in Table 2.

Table 3 portrays the slow neuromuscular recruitment of TA on EO FIRM (control, $6.2 \pm 4.3 \mathrm{~s}$; HIV, $10.01 \pm 5.9 \mathrm{~s}$ ), and EC HUD (control, $8.02 \pm 5.7 \mathrm{~s}$; HIV, $11.8 \pm 5.1 \mathrm{~s}$ ) when comparing the HIV and control groups. Likewise, the TA indicated significantly slower recruitment $(p<0.05)$ exhibited by an extended time to peak activation (control, 0.44 $\pm 0.38 \mathrm{~s}$; HIV, $0.78 \pm 0.52 \mathrm{~s}$ ) during EC while standing on the foam.

Additionally, a significant increase in muscle activation $(p<0.05)$ was observed (Table 2$)$ by increased decay (control, $0.61 \pm 0.36 \mathrm{~s} ; \mathrm{HIV}, 0.91 \pm 0.72 \mathrm{~s}$ ) in the GA and duration (control, $1.03 \pm 0.47 \mathrm{~s}$; HIV, $1.6 \pm 0.99 \mathrm{~s}$ ) in the TA (Table 3) during EC on an unstable surface.

During the most complex single tasks, EC HUD FOAM, in addition to a significantly $(p<0.05)$ prolonged onset (control, $8.02 \pm 5.7 \mathrm{~s} ; \mathrm{HIV}, 11.8 \pm 5.1 \mathrm{~s}$ ) of the TA, the HIV group exhibited a significantly prolonged activation $(p<0.05)$ by increasing decay time (control, $0.49 \pm 0.22 \mathrm{~s} ; \mathrm{HIV}, 0.55 \pm 0.31 \mathrm{~s}$ ).

When comparing muscle recruitment timing during the dual-cognitive tasks, the results were comparable between groups $(p>0.05)$ for both the GA and TA.

\section{Discussion}

This study sought to determine lower limb neuromuscular recruitment activation patterns while standing among physically active PLHIV in comparison to HIV-negative, physically active individuals.

We hypothesized that, in comparison to the control group, PLHIV would exhibit evident neuromuscular recruitment alterations in the TA and GA muscles during standing postural control tasks. Our hypothesis was corroborated by our findings of slower neuromuscular recruitment alteration and prolonged activity in four out of five single balance tasks 
Table 3. Electromyographic onset, time to peak, duration, and decay of tibialis anterior during balance tasks. Results of multivariate analysis of variance (MANOVA) performed between asymptomatic HIV group and control group. Significance level set at $p \leq 0.05$

\begin{tabular}{|c|c|c|c|}
\hline Task/Variables (s) & $\begin{array}{l}\text { Control } \\
(n=19)\end{array}$ & $\begin{array}{l}\text { Asymptomatic } \\
\text { HIV }(n=18)\end{array}$ & $p$-value \\
\hline \multicolumn{4}{|l|}{ Tibialis anterior } \\
\hline \multicolumn{4}{|l|}{ EO Firm } \\
\hline Onset & $6.20 \pm 4.30$ & $10.01 \pm 5.90$ & 0.01 \\
\hline Time to peak & $0.49 \pm 0.42$ & $0.67 \pm 0.52$ & 0.16 \\
\hline Duration & $0.99 \pm 0.30$ & $1.05 \pm 0.50$ & 0.71 \\
\hline Decay & $7.20 \pm 4.40$ & $11.20 \pm 5.90$ & 0.41 \\
\hline \multicolumn{4}{|l|}{ EO Foam } \\
\hline Onset & $8.07 \pm 3.90$ & $7.90 \pm 4.80$ & 0.91 \\
\hline Time to peak & $0.50 \pm 0.68$ & $0.43 \pm 0.33$ & 0.52 \\
\hline Duration & $1.10 \pm 0.99$ & $0.02 \pm 0.64$ & 0.68 \\
\hline Decay & $0.59 \pm 0.43$ & $0.59 \pm 0.43$ & 0.98 \\
\hline \multicolumn{4}{|l|}{ EC Foam } \\
\hline Onset & $8.60 \pm 5.20$ & $8.07 \pm 4.60$ & 0.69 \\
\hline Time to peak & $0.44 \pm 0.38$ & $0.78 \pm 0.52$ & 0.005 \\
\hline Duration & $1.03 \pm 0.47$ & $1.60 \pm 0.99$ & 0.005 \\
\hline Decay & $0.58 \pm 0.32$ & $0.80 \pm 0.66$ & 0.09 \\
\hline \multicolumn{4}{|l|}{ EO HUD } \\
\hline Onset & $8.30 \pm 4.30$ & $10.01 \pm 6.4$ & 0.24 \\
\hline Time to peak & $0.47 \pm 0.27$ & $0.44 \pm 0.34$ & 0.66 \\
\hline Duration & $0.94 \pm 0.43$ & $0.98 \pm 0.77$ & 0.83 \\
\hline Decay & $0.47 \pm 0.29$ & $0.54 \pm 0.51$ & 0.50 \\
\hline \multicolumn{4}{|l|}{ EC HUD } \\
\hline Onset & $8.02 \pm 5.70$ & $11.80 \pm 5.10$ & 0.05 \\
\hline Time to peak & $0.47 \pm 0.26$ & $0.63 \pm 0.39$ & 0.06 \\
\hline Duration & $0.95 \pm 0.35$ & $1.18 \pm 0.53$ & 0.06 \\
\hline Decay & $0.49 \pm 0.22$ & $0.55 \pm 0.31$ & 0.05 \\
\hline \multicolumn{4}{|l|}{ EO COG } \\
\hline Onset & $9.60 \pm 4.40$ & $12.10 \pm 5.20$ & 0.06 \\
\hline Time to peak & $0.47 \pm 0.58$ & $0.59 \pm 0.53$ & 0.47 \\
\hline Duration & $1.05 \pm 1.10$ & $1.06 \pm 0.75$ & 0.97 \\
\hline Decay & $0.57 \pm 0.58$ & $0.47 \pm 0.38$ & 0.49 \\
\hline \multicolumn{4}{|l|}{ EC COG } \\
\hline Onset & $8.70 \pm 5.10$ & $8.20 \pm 4.80$ & 0.73 \\
\hline Time to peak & $0.46 \pm 0.24$ & $0.43 \pm 0.29$ & 0.67 \\
\hline Duration & $0.98 \pm 0.48$ & $0.86 \pm 0.41$ & 0.37 \\
\hline Decay & $0.52 \pm 0.35$ & $0.43 \pm 0.27$ & 0.36 \\
\hline
\end{tabular}

exhibited among both the TA and GA in the HIV group. Therefore, we accept our hypothesis.

Various factors have the ability to impact gait and balance in PLHIV; therefore, this work defined neuromotor alter-
Table 3. Cont.

\begin{tabular}{|c|c|c|c|}
\hline Task/Variables (s) & $\begin{array}{l}\text { Control } \\
(n=19)\end{array}$ & $\begin{array}{l}\text { Asymptomatic } \\
\text { HIV }(n=18)\end{array}$ & $p$-value \\
\hline \multicolumn{4}{|l|}{ Gastrocnemius } \\
\hline \multicolumn{4}{|l|}{ EO HUD COG } \\
\hline Onset & $9.60 \pm 4.70$ & $8.90 \pm 4.70$ & 0.63 \\
\hline Time to peak & $0.58 \pm 0.31$ & $0.65 \pm 0.47$ & 0.51 \\
\hline Duration & $1.20 \pm 0.67$ & $1.21 \pm 0.77$ & 0.69 \\
\hline Decay & $0.62 \pm 0.53$ & $0.57 \pm 0.40$ & 0.95 \\
\hline \multicolumn{4}{|l|}{ EC HUD COG } \\
\hline Onset & $8.30 \pm 5.60$ & $8.50 \pm 5.60$ & 0.90 \\
\hline Time to peak & $0.44 \pm 0.29$ & $0.54 \pm 0.37$ & 0.27 \\
\hline Duration & $1.00 \pm 0.45$ & $1.10 \pm 0.52$ & 0.37 \\
\hline Decay & $0.56 \pm 0.33$ & $0.58 \pm 0.42$ & 0.84 \\
\hline
\end{tabular}

ations as characteristics affecting the normal motor function for PLHIV. In their study, Berner et al. [6] described distinct parameters causing neuromotor alterations in PLHIV. Moreover, researchers have observed that PLHIV displayed more incidence of decreased walking speed, increased sway, and inadequate postural reflexes when compared to non-HIV subjects. These neuromotor alterations are clear during challenging conditions, such as fast walking [6] and challenging balance tasks, such as eyes closed with head movements while standing [16]. It has been postulated that these neuromotor alterations are associated with the impact of hardships stemming from HIV disease itself, and not the direct impact of ART medications on the central nervous system (CNS) [6].

Although they were physically active, the BMIs of the HIV-group participants in this study were above average (overweight, 25.1 \pm 5.7 ), suggesting that weight may be considered as one of the causes for a neuromuscular recruitment alteration. Bauer et al. [7] discussed the incidence of adverse effects of HIV/AIDS on balance and gait when a person's body mass increases. The authors assumed that HIV was associated with delayed gait initiation time and reduced gait speed, with an intriguing finding being that HIV-positive individuals with a BMI categorized as obese displayed more impairments in cadence and gait onset time in comparison to those with a lower BMI index, including frail adults. We believe the delayed onset of muscle activation observed during standing balance with the HIV group related to the previous findings. PLHIV, as a consequence of ART, could suffer from hyperlipidemia and Buffalo's hump [13], two afflictions that potentially increase their BMI and alter postural control. Therefore, the previous outcome has clinical implications as far as emphasizing the need for diet, exercise, and balance therapy among patients living with HIV [7].

Another reason that the neuromuscular pattern in PLHIV is modified might be related to an altered posture reflex and gait deficiencies. In their systematic review, Berner et al. [6] found that decreased walking speed, increased 
sway, and impaired postural reflexes were the main neuromotor alterations in PLHIV. Their report suggests that these impairments were more pronounced during challenging conditions, such as fast walking or when eyes were closed during static balance, and have more of a correlation with the direct effects of HIV on the central nervous system than to the ART medications used to treat the disease [6].

Disruptions on a more physiological and cellular level could pose as the cause for our study's findings, as postulated by Berner et al. [6]. In their investigation, the authors suggested various rationales for how HIV induces balance impairments, with one conception being that the medications, which deteriorate mitochondria, in turn distort muscle function, leading to sarcopenia and osteoporosis [6]. Decreased lower extremity strength results in altered activation of the leg musculature, causing a shift from ankle strategy to a hip strategy and increased postural sway.

In their study, Rosario et al. [16] explained an unusual increase in sway with normal muscle activation in HIV participants when compared to participants in the control group. Additionally, the authors suggested that this increase in postural instability was associated with proximal components on a kinematic chain, such as the hip, instead of lower leg muscle weakness and ankle strategies [16]. Researchers have agreed that the lower limb muscle weakness was a component for balance difficulties, and still, they added the reduction of muscle power as one of the variables heightening neuromotor alteration in PLHIV [12]. In the present study, the findings illustrated an alteration of muscle recruitment that can be explained by a reduction in muscle power of the TA and GA.

Our results imply that the emergence of these compensatory strategies stem from an impaired balance sensory system, which was evidently displayed by the altered GA and TA muscle recruitment in the HIV group. Instead of the brain processing accurately proprioceptive and vestibular inputs during challenging activities and overriding the inaccurate visual system, disturbance occurs, resulting in compensation through changing the GA and TA recruitment pattern. Another possibility for the outcome of this study is a miscommunication between the central and peripheral nervous systems.

We suggest a neuromuscular assessment and evaluation of a muscle recruitment in all stages of HIV, even among physically active individuals, in order to gain a greater comprehension of the lower limb neuromuscular activation mechanism and its role on standing posture. Although physically active PLHIV had better compensatory strategies than sedentary PLHIV, the disease still has the ability to cause balance impairments among these individuals [6]. The participants in this study were physically active and had their HIV condition controlled (CD4 count > 200), and but still, neuromuscular modifications were found.

The second intent of this study was associated with the cognitive aftermath of HIV's impact on balance. The current study explored how adding cognitive-dual tasks modify lower limb muscle recruitment during standing balance in PLHIV. We hypothesized that as the balance tasks increase in complexity and incorporate dual-cognitive tasks, modifications in the neuromuscular alteration recruitment pattern of the tibialis anterior and gastrocnemius muscles would increase. However, our results showed that both the HIV group and control group had comparable muscle recruitment during the dual-cognitive tasks. Therefore, we reject our hypothesis.

Some neurocognitive alterations that PLHIV are suffering from are dementia, anxiety, and depression [5]. When specifically investigating the Hispanic-Latino Puerto Rican population, the principal reported comorbidity was depression, followed by peripheral neuropathy [15]. These neurocognitive changes should be crucial when taken into consideration, as these disorders considerably pave the path for a cognitive decline, decreased quality of life, and reduced physical activity as well as being at an increased risk of falling and having a fear of falling $[8,9]$.

In their systematic review, Banks et al. [5] observed that among PLHIV who were 50 years of age and above, more than a half reported a fall in the last year, and approximately one third reported having difficulties with balance. It was the intention of the present study to understand the mechanisms related to neuromuscular control and balance impairments in PLHIV. For that purpose, we examined standing posture, neuromotor alteration, and the role of split attention by adding a cognitive component to multiple static balance tasks. Astonishingly, the muscle recruitment of the lower limb musculature demonstrated by the HIV group was similar to those of the control group.

An additional effort of this research was to establish our assumptions from previous studies regarding the occurrence of neurocognitive alterations and the increase in postural sway during dual-cognitive tasks among individuals living with HIV $[5,16]$. Although PLHIV present higher rates of dementia, anxiety, and depression, those are factors, which separate from neuromuscular modification in the HIV group $[5,8,9]$. The neurocognitive difficulties mentioned above are indisputably contributing to the increased fall risk, increased fear of falling, decreased physical activity, and cognitive decline; however, they are unrelated to our study and our findings.

Several factors can explain these previous results. Firstly, none of the HIV participants from this study had a peripheral neuropathy diagnosis. Secondly, the participants of this study were physically active people; there is an evidence that exercise can ameliorate the muscle weakness associated with HIV complications [14]. Finally, the participants in this study were asymptomatic, did not have a history of falling, and had their HIV disease under control. Nonetheless, irrespective of the physical profile of people living with HIV, from our findings, we strongly recommend a neuromuscular assessment regardless of the subject's status or medical history.

\section{Conclusions}

The intention of this investigation was to explore the mechanism behind lower extremity muscle recruitment in people living with HIV. In understanding this distinct muscle activation mechanism and pattern, clinicians involved in the rehabilitation and prevention of HIV difficulties will be able 
to develop more targeted intervention plans tailored to their patients' needs. This inquiry illustrates the identification of the muscle activation recruitment modifications in both the gastrocnemius and tibialis anterior muscles in physically active HIV-positive individuals, who have no fall history and are otherwise healthy. The findings from this work indicate the elemental necessity of incorporating neuromuscular examinations of the lower limbs into fitness programs for all those being treated for HIV. This study targeted the ankle complex and the gastrocnemius and tibialis anterior, which are the primary muscles responsible for anterior and posterior sway as well as ankle strategies. Given the importance of proximal lower extremity musculature modifications among those living with HIV, the examination of the gastrocnemius and tibialis anterior muscles in this investigation remains a drawback of this framework. We suggest that placing emphasis on the proximal musculature related to the hip and hip strategies should be assessed, such as gluteus maximus. Finally, we recommend the examination of neuromuscular recruitment of the lower limbs among HIV-positive individuals in different stages of the disease, in addition to those who are inactive and who have been diagnosed with AIDS.

\section{Conflict of interest}

The author declares no conflict of interest with respect to the research, authorship, and/or publication of this article.

\section{References}

1. Centers for Disease Control and Prevention. Estimated HIV incidence and prevalence in the United States 2010-2016. 2019. Available at: https://www.cdc.gov/hiv/pdf/library/reports/surveillance/cdc-hiv-surveillance-supplemental-report-vol-24-1.pdf

2. Erlandson KM, Allshouse AA, Jankowski CM, et al. Comparison of functional status instruments in HIV-infected adults on effective antiretroviral therapy. HIV Clin Trials 2012; 13: 324-334.

3. Cohen HS, Cox C, Springer G, et al. Prevalence of abnormalities in vestibular function and balance among HIV-seropositive and HIVseronegative women and men. PLoS One 2012; 7: e38419.

4. Heinze B, Swanepoel DW, Hofmeyr LM. Systematic review of vestibular disorders related to human immunodeficiency virus and acquired immunodeficiency syndrome. J Laryngol Otol 2011; 125: 881-890.

5. Banks LM, Zuurmond M, Ferrand R, Kuper H. The relationship between HIV and prevalence of disabilities in sub-Saharan Africa: a systematic review. Trop Med Int Health 2015; 20: 411-429.

6. Berner K, Morris L, Baumeister J, Louw Q. Objective impairments of gait and balance in adults living with HIV-1 infection: a systematic review and meta-analysis of observational studies. BMC Musculoskelet Disord 2017; 18: 325.

7. Bauer LO, Wu Z, Wolfson LI. An obese body mass increases the adverse effects of HIV/AIDS on balance and gait. Phys Ther 2011; 91: 1063-1071.

8. Shah KN, Majeed Z, Yoruk YB, et al. Enhancing physical function in HIV-infected older adults: a randomized controlled clinical trial. Health Psychol 2016; 35: 563-573.

9. Veeravelli S, Najafi B, Marin I, Blumenkron F, Smith S, Klotz SA. Exergaming in older people living with HIV improves balance, mobility and ameliorates some aspects of frailty. J Vis Exp 2016; 116: 54275 .
10. Schrack JA, Althoff KN, Jacobson LP, et al. Accelerated longitudinal gait speed decline in HIV-infected older men. J Acquir Immune Defic Syndr 2015; 70: 370-376.

11. Oliveira VH, Wiechmann SL, Narciso AM, Webel AR, Deminice R. Muscle strength is impaired in men but not in women living with HIV taking antiretroviral therapy. Antiviral Ther 2018; 23: 11-19.

12. Richert L, Dehai P, Mercié P, et al.; Groupe d'Epidémiologie Clinique du SIDA en Aquitaine (GECSA). High frequency of poor locomotor performance in HIV-infected patients. AIDS 2011; 25: 797-805.

13. Ceccarelli G, d'Ettorre G, Marchetti F. Development of Buffalo Hump in the course of antiretroviral therapy including raltegravir and unboosted atazanavir: a case report and review of the literature. J Med Case Rep 2011; 5: 70 .

14. Unhjem R, Nygård M, Hoven LTVD, Sidhu SK, Hoff J, Wang E. Lifelong strength training mitigates the age-related decline in efferent drive. J Appl Physiol 2016; 121: 415-423.

15. Rosario MG, Jamison L, Gines G. The role of HIV antiretroviral medication on motor-cognitive and neurological alterations in Hispanic people living with HIV. J Pub Health Issue Pract 2020; 4: 160.

16. Rosario MG, Marshall J, Herkert A, Binoy B, Windham H, Orozco E. Lower limb neuromuscular modification and standing postural control alteration in apparent asymptomatic people living with HIV. J Rehab Pract Res 2020; 1: 102. 